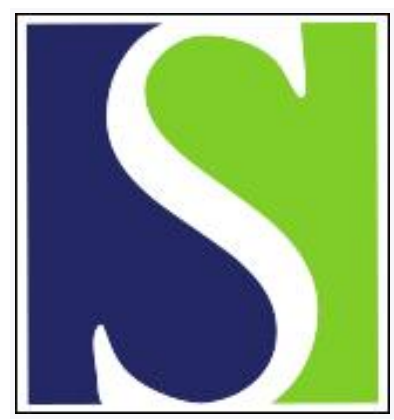

Scand J Work Environ Health 2002;28(1):58-63

https://doi.org/10.5271/sjweh.647

Issue date: Feb 2002

Alternative for estimating the burden of lung cancer from occupational exposures - some calculations based on data from Swedish men

by Axelson 0

Affiliation: Division of Occupational and Environmental Medicine, Department of Health and Environment, Linköping University, SE-58185 Linköping, Sweden. olav.axelson@ymk.liu.se

Refers to the following texts of the Journal: 2001;27(3):161-213 1999;25 suppl 2:1-116

The following article refers to this text: $2013 ; 39(1): 106-111$

Key terms: burden of lung cancer; confounding; epidemiologic model; incidence; man; occupational exposure; sensitivity; smoking; Sweden

This article in PubMed: www.ncbi.nlm.nih.gov/pubmed/11871854 


\title{
Alternative for estimating the burden of lung cancer from occupational exposures - some calculations based on data from Swedish men
}

\author{
by Olav Axelson, MD ${ }^{1}$
}

\begin{abstract}
Axelson 0. Alternative for estimating the burden of lung cancer from occupational exposures - some calculations based on data from Swedish men. Scand J Work Environ Health 2002;28(1):58-63.

Objectives This study attempts to demonstrate a calculation of the occupational lung cancer burden using economically active men in Sweden as an example.

Methods Estimates were calculated using Swedish register data on occupation in 1970, lung cancer incidence in 1971-1989, smoking frequencies in 1963, and the formula $\mathrm{I}=\mathrm{RI}_{0} \mathrm{~F}+\mathrm{I}_{0}(1-\mathrm{F})$, where $\mathrm{I}$ is the overall incidence, $\mathrm{R}$ is the relative risk associated with a factor (here smoking), $\mathrm{F}$ is the fraction of persons at risk (smokers), and $\mathrm{I}_{0}$ is the incidence among those not at risk (nonsmokers).

Results Farmers, gardeners, forestry workers, and fishermen had the lowest lung cancer risk (42.1 per 100000 person-years) and a smoking frequency of $44.7 \%$. Their $\mathrm{I}_{0}$ was 12.6 or 8.4 per 100000 person-years, taking $\mathrm{R}$ for smoking as 6 or 10, respectively. From these $\mathrm{I}_{0}$ estimates, the expected rates for white- and blue-collar workers (smoking frequencies 52.7 and $57.7 \%$, respectively) were 45.8 and 49.1 per 100000 person-years, as compared with the $22 \%$ and $57 \%$ higher observed rates, respectively. Weighing these excesses proportionally according to the sizes of the three occupational categories gave, respectively for R equal to 6 and 10, occupation-related excesses of $39 \%$ and $32 \%$ and population-attributable risks of $28 \%$ and $24 \%$.

Conclusions About one-fourth of the lung cancers that occur among economically active Swedish men seem to have been related to occupation. This figure agrees with estimates made by other methods in Nordic countries. Due to interaction, the population-attributable risk from smoking is still high, $73 \%$ and $83 \%$ at relative risk values of 6 and 10, respectively.
\end{abstract}

Key terms confounding, epidemiologic model, incidence, sensitivity, smoking.

It is generally accepted that smoking is the primary risk factor for lung cancer. The contribution of occupational exposures is also well recognized and has been evaluated from time to time with different models. A casereferent approach considering jobs with known or suspected exposure to carcinogens is one such model. Another has been to apply available risk estimates for specific exposures or jobs to the occurrence of these factors in the population (1). Cancer risks by occupation have also been studied, for example, through use of register data in the Nordic countries (2). Some of the register data are used in this paper to demonstrate an alternative for estimating the overall impact of occupational exposures on lung cancer in a population. This model can also be subjected to sensitivity analyses to illustrate the potential tenability of an estimate, let alone the inherent approximations.

\section{General aspects of occupational lung cancer}

Smoking is usually considered to be the most important confounding factor in studies of occupational cancer and, in particular, lung cancer. Investigations in many countries have shown that smoking is more prevalent among blue-collar workers than among persons in other sectors of the population. Accordingly, any observed excess of lung cancer among blue-collar workers is assumed to be related not only to occupational exposures, but also to a higher rate of smoking. This view is rather widely accepted and emphasized, especially when direct information on smoking is lacking in any particular study.

Interaction phenomena regarding lung cancer risks have also been reported. A classical example is the strong synergistic effect of smoking and asbestos

Division of Occupational and Environmental Medicine, Department of Health and Environment, Linköping University, Linköping, Sweden.

Reprint requests to: Professor Olav Axelson, Division of Occupational and Environmental Medicine, Department of Health and Environment, Linköping University, SE-581 85 Linköping, Sweden. [E-mail: olav.axelson@ymk.liu.se] 
exposure on lung cancer (3). Similarly, arsenic and smoking act synergistically to increase the risk of lung cancer (4), and such syngerism also seems to occur for smoking and exposure to radon progeny, although the results in the literature vary (5). There are additional examples of synergistic effects that should not be confused with confounding, especially as synergistic phenomena can be utilized in the context of preventive measures.

The influence of uncontrolled smoking and various other potential confounders on risk estimates can be calculated, albeit crudely, given some knowledge of the strength and occurrence of these factors (6). To assess the contribution of aggregated occupational exposures to the risk of lung cancer in all different branches, this type of calculation was done using register-based data on Swedish men aged 25-64 years and economically active at the time of the census in 1970 (2). However, as in other studies evaluating the occupational burden of lung cancer, the applied model failed to adjust for widespread risk factors other than smoking, for instance, exposure to indoor radon, air pollution from traffic, and other exposures in urbanized and industrialized areas.

\section{Subjects and methods}

The probable magnitude of the influence of smoking on the risk estimates for the population was calculated using the formula $\mathrm{I}=\mathrm{RI}_{0} \mathrm{~F}+\mathrm{I}_{0}(1-\mathrm{F})$, where $\mathrm{I}$ is the overall incidence, $\mathrm{R}$ is the relative risk associated with a factor (in this case smoking), $\mathrm{F}$ is the fraction of persons at risk (smokers), and $\mathrm{I}_{0}$ is the incidence among those not at risk (nonsmokers) (6). Several terms can be added to the formula, given information on relative risks and exposure frequencies for different categories of the factor.

There are different and fairly divergent estimates of the overall risk of lung cancer from smoking in the literature. In a study from southeastern Sweden the relative risk from smoking was found to be 5.1 for male and female smokers together (7). In a nationwide Swedish study of indoor radon and lung cancer, the published data permit the calculation of an overall crude relative risk to be 6.0 for males and females combined (8). Moreover, relative risk values of 9.6, 5.9, and 2.7 have been reported to correspond to smoking more than 15 grams of tobacco per day, $8-15$ grams per day, and less than 8 grams per day, respectively (9). The estimates from the cited studies suggest a relative risk of 6 as a reasonable average for smokers in Sweden. Relative risks for male and female smokers may be similar (10) or slightly higher for women (11), thus a value of 6 may even be somewhat high. If that is the case, the following calculations are somewhat conservative. To illustrate the sensitivity of the model on a more extreme level, I also used an average relative risk of 10 in my calculations.

For the age group 18-49 years in 1963 in Sweden, $57.8 \%$ of the blue-collar workers and $52.7 \%$ of the white-collar workers (including professionals such as technical workers and laboratory assistants) were smokers, whereas the corresponding value for farmers, fishermen, and forestry workers was $44.7 \%$ (12). These persons were 25-56 years old in 1970, which is the year a cohort aged 25-64 years was chosen from census data (2) and which was used for my calculations. The 1970 census also provided information on occupation and the follow-up of the cohort covered 1971-1989. The cancer cases occurring during this period were retrieved from the Swedish cancer register, which is based on compulsory registration. Additional background information for the calculations is given in table 1 .

\section{Results}

When the crude incidence rate of 42.1 cases per 100000 person-years for lung cancer among farmers (including gardeners), fishermen, and forestry workers (for simplicity, collectively referred to as "outdoor workers" in the rest of this article) was used as a basis and a relative risk of 6 was assumed, the lowest expected nonsmoker lung cancer rate was 12.6 per 100000 person-years. From the data in table 1, along with the calculated baseline rate for nonsmokers, and again an average relative risk from smoking of 6 , predicted smoking-adjusted rates were calculated for the white- and blue-collar

Table 1. Population data on smoking and lung cancer in 1971-1989 $(2,12) .^{\text {a }}$

\begin{tabular}{|c|c|c|c|c|}
\hline Population group & $\begin{array}{l}\text { Number of } \\
\text { lung cancers }\end{array}$ & Person-years & $\begin{array}{l}\text { Crude incidence per } \\
10^{5} \text { person-years }\end{array}$ & $\begin{array}{l}\text { Smoking frequency (\%) } \\
\text { in } 1963 \text { in the 18- to } \\
49 \text {-year age group }\end{array}$ \\
\hline White-collar workers & 5964 & 10692381 & 55.77 & 52.7 \\
\hline $\begin{array}{l}\text { Farmers, gardeners, fishermen, } \\
\text { forestry workers }\end{array}$ & 1291 & 3067958 & 42.08 & 44.7 \\
\hline Blue-collar workers & 13070 & 16968799 & 77.02 & 57.8 \\
\hline
\end{tabular}

a Only economically active men aged 25-64 years in 1970 were taken into consideration. There were 3433 lung cancers in 3014234 person-years for economically inactive persons, with a crude rate of 70.40 per $10^{5}$ person-years. 
workers. The predicted rates were 45.8 and 49.1 per 100000 person-years, respectively, as compared with the observed crude rates of 55.8 and 77.0 per 100000 person-years. Formally, the excess relative risk for white-collar workers was $55.8 / 45.8-1=0.22$, indicating an occupation-related lung cancer burden of $22 \%$. The corresponding value for blue-collar workers was $57 \%$, whereas no occupational excess was ascribed to the category comprising outdoor workers. Weighing these estimates together with regard to the relative sizes of the three populations indicated that an excess of $39 \%$ of the lung cancers could be attributed to occupation (including environmental exposures in industrialized areas and determinants other than smoking that may be associated with hazardous occupational exposures). The corresponding population-attributable risk was 28\% (39/139). However, this weighing did not take into account the economically inactive men (table 1).

On the assumption of a relative risk of 10.0 from smoking, instead of 6.0, the baseline rate for nonsmokers was 8.4 per 100000 person-years, and the excess burden was $16 \%$ for white-collar workers and $48 \%$ for blue-collar workers. The weighted overall burden was then $32 \%$, and the population-attributable risk was $24 \%$. Relative risks of 6.0 and 10.0 in the calculations correspond to attributable risks of $83 \%$ and $90 \%$, respectively, for the smokers and population-attributable risks of $73 \%$ and $83 \%$, respectively.

\section{Discussion}

According to my estimations for active Swedish men, an excess of $39 \%$ or $32 \%$ of the lung cancer burden in the recent past should be attributable to various occupational exposures. The model used may appear to be too simple. However, it has been applied in parallel with three other, more elaborate models to estimate the lung cancer mortality rates in Italy not attributable to smoking, and the results obtained were fairly similar (13). The three other models also included dose in terms of number of cigarettes smoked, as well as duration of smoking, along with linear and exponential terms. In the cited study (13), the background lung cancer rate for nonsmokers was about 9-10 cases per 100000 personyears, which is essentially comparable to the estimates obtained by me.

Clearly, an evaluation of this kind is influenced by secular trends in occupational mobility, smoking habits, and cancer risks. According to the report (2) underlying the present calculations, $45.5 \%$ of Swedish men were registered as having the same type of job in both the 1960 and the 1970 census. Nonetheless, it is likely that the turnover between the three main groups considered here was less pronounced, especially between the white-collar workers and the other two categories. However, some of the outdoor workers probably became blue-collar workers and were accordingly exposed to the somewhat increased risk of lung cancer risk associated with that category. Therefore, the baseline lung cancer incidence would have been biased upwards and would have had a conservative effect on the final estimates of the occupational lung cancer burden.

Neglecting ex-smokers in this evaluation would have had the same conservative effect, because these persons would fall into the category of nonsmokers and thereby decrease the relative risk for the smokers in comparison with the relative risks of 6 and 10 in my calculations. As indicated from the use of 6 and 10 as relative risks, a lower relative risk for smoking would lead to a higher estimate of the occupationally related burden of lung cancer. Another source of underestimation of the occupational lung cancer burden is the fact that smoking declined during the follow-up period from which the underlying data were obtained (2). Due to this decline, the percentages used in the calculations to represent smokers during the follow-up period 1971-1989 actually represented not only smokers, but also an increasing number of ex-smokers. This phenomenon would have reduced the true average relative risk of lung cancer for the "original" smokers, that is, those accounted for by the percentages used in the calculations, so that the used estimates of 6 and 10 could be thought of as overestimates.

The group of economically inactive men comprised $17 \%$ of all lung cancers (and the incidence was relatively high; see table 1) but could not be included in the calculated estimates due to a lack of relevant data regarding earlier employment. Nonetheless, this group probably comprised a relatively large number of persons with backgrounds as blue-collar workers, as well as many people who had never been employed and were thereby similar to white-collar workers in regard to a lack of hazardous occupational exposures. Therefore, the contribution of occupational exposures to the risk of lung cancer in this economically inactive group may have been fairly similar to the estimates calculated for the economically active men.

My estimations of the excess occupational risk of lung cancer among Swedish men are comparable with the results of a study conducted in Norway (14). Despite its case-referent approach, the Norwegian evaluation indicated a population-attributable risk of $22-35 \%$ when occupations entailing definitely hazardous exposures were considered, and the estimate rose to $37-47 \%$ when jobs with "possibly exposed" categories were also included. Asbestos exposure was the most important single risk factor in that study, but the estimated 
contribution of smoking was nonetheless $82 \%$, which is similar to my results obtained using a value of 10 as the average relative risk for smokers. The indicated estimates are in no way contradictory because attributable risks add up to more than $100 \%$ when there is interaction between the risk factors. In another Norwegian study (15), definite exposure to occupational carcinogens was estimated to be involved in $13-27 \%$ of the cases, and a fairly similar estimate of $29 \%$ for men has recently been reported from Finland (1). A predictive study regarding avoidable cancers in the Nordic countries suggested that, for Swedish men, $22 \%$ of the lung cancers (505 out of 2299) in the year 2000 would depend on industrial exposures to arsenic, polyaromatic hydrocarbons, metals, and mining (16). Furthermore, these authors, in another article (17), indicated that smoking in 1980, 1990, and 2000 would be responsible for, respectively, $83 \%, 83 \%$, and $81 \%$ of the lung cancer cases diagnosed among Swedish men; this finding is similar to the aforementioned Norwegian estimate and also agrees fairly well with my calculations.

In studies of known hazardous exposures in various types of jobs, it has earlier been suggested that occupational exposures account for as little as 5\% to as much as $40 \%$ of the lung cancer in some populations $(1,7$, 18-21) (table 2). Considering only a few important and widespread exposures, a Swedish study (22) indicated an attributable risk of $9.5 \%$ for exposure to asbestos, diesel exhaust, and other combustion products, whereas the inclusion of a somewhat broader spectrum of occupational risk factors resulted in the aforementioned estimate of $22 \%$ (16). However, in such selective evaluations, various hazardous exposures operate among the unexposed and thus reduce the relative risk for the exposed along with the attributable risk. In contrast, the present model is not only countrywide but also inclusive of hitherto unrecognized occupational cancer risks that may fall in the unexposed category when other approaches are used as leading to an "unclean" reference entity with an increased incidence. Consequently the relative risk estimate is too low and causes an underestimation of the occupational risk burden even among those exposed to recognized hazardous exposures.
Kunst and his co-workers (23) calculated the ratio of lung cancer mortality for manual to nonmanual workers in several European countries and obtained the following values: 2.20 for Finland, 1.65 for France, 1.62 for Norway, 1.54 for England and Wales, 1.51 for Denmark, 1.46 for Sweden, and 1.07 for Portugal (23). In my study, based on the incidence data published by Andersen et al (2), the lung cancer incidence rates were 77.0 and 55.8 cases per 100000 person-years for bluecollar and white-collar workers, respectively, giving a ratio of 1.38 , which is slightly conservative when compared with the mortality ratio of 1.46 that Kunst et al reported for Sweden.

An extreme minimum lung cancer burden from occupational exposures can be calculated from Swedish data on smoking and lung cancer risk in different occupations (9). More specifically, standardized incidence ratios (SIR), as well as smoking-adjusted such ratios for lung cancer, were, respectively, 0.44 and 0.48 for teachers but 1.81 and 1.53 for miners and quarrymen. The corresponding ratios were 0.44 and 0.70 for farmers and 0.45 and 0.61 for farm workers. Many manual worker groups had SIR values around 1.20, and the smokingadjusted estimates were about $10 \%$ lower. For the Swedish men aged 20-69 years included in that study, the proportion of regular smokers ranged from $24 \%$ to $68 \%$ in the various occupational groups, but neither teachers nor miners and quarrymen had the most extreme smoking habits.

A low extreme contribution from occupational exposures can be estimated by dividing the average national SIR, defined as 1.00 , by the lowest reported SIR of 0.44 , which gives a value of 2.27 . When the observed extremes for smoking, $24 \%$ and $68 \%$, were taken into account along with a relative risk of 6.0 for smoking and the formula already presented was used, the confounding relative risk was 2.0. However, this estimate of the influence of smoking should be extremely high with regard to the underlying maximized difference in smoking habits. Even after adjustment for such a strong and clearly exaggerated confounding effect, the excess relative risk is $2.27 / 2.0-1=0.14$, indicating an occupational contribution of $14 \%$ (or a $12 \%$

Table 2. Examples of estimates of the contribution from occupational exposures to the occurrence of lung cancer.

\begin{tabular}{llll}
\hline Reference & Country or area & Estimate (\%) & Remarks \\
\hline Doll \& Peto, $1981(18)$ & United States & $15 / 5$ & Men/women \\
Pastorino et al, $1984(20)$ & Northern Italy & 33 & Men \\
Kjuus et al, $1986(14)$ & Norway & $22-47$ & Men \\
Kvale et al, $1986(15)$ & Norway & $13-27$ & Men \\
Damber \& Larsson, 1987 (19) & Northern Sweden & $9-18$ & Men \\
Dave et al, 1988 (7) & Southern Sweden & 8 & Both genders \\
Ronco et al, $1988(21)$ & Northern Italy & $12-36$ & Men \\
Nurminen \& Karjalainen, 2001 (1) & Finland & $29 / 5$ & Men/women \\
\hline
\end{tabular}


population-attributable risk). The estimate derived in this manner should represent a more or less unlikely extreme minimum contribution from occupational factors.

The extremity of the indicated estimate can be elucidated by data from Finland reported by Asp (24). In that study, health screening conducted from 1970 to 1975 revealed that $51 \%$ of farmers and animal tenders were current and former smokers, civil servants coming next with 59\% ever smokers. At the other extreme, $90 \%$ of construction workers and $93 \%$ of machine operators had ever smoked. On the assumption of a relative risk of 10 for former smokers and 20 for current smokers, the confounding relative risk was 0.67 for civil servants, 1.28 for machine operators, and 1.31 for construction workers. Consequently, for these extreme groups, the smoking differences almost doubled the risk to $1.31 / 0.67=1.96$, which is similar to the aforementioned confounding relative risk of 2.0 as already calculated on the basis of Swedish data (9).

With the use of the value of 2.20 that Kunst et al (23) obtained as the ratio of manual to nonmanual workers in regard to relative risk for lung cancer in Finland, the excess risk of lung cancer would be 2.20/1.96$1=0.12$. Again, this excess contribution of $12 \%$ (population-attributable risk of $11 \%$ ) from occupational and general exposures other than smoking represents an extreme estimate of the occupational lung cancer burden based on the occupational groups exhibiting the most divergent smoking habits.

In conclusion, it seems reasonable to assume that, for men, about a fourth or more of the lung cancer burden in Sweden during the later part of the previous century was related to occupational exposures. Data from both Norway and Finland corroborate this assumption $(1,14,15)$. All the indicated estimates also include effects of life-style factors other than smoking, as well as environmental exposures associated with living and working in industrialized areas. The impact of such background factors should be relatively weak, however, as compared with the effect of smoking and industrial exposures. Furthermore, a very strong association of such other factors with the hazardous occupational exposures would be required for any substantial confounding to arise in this respect. Even under the most extreme assumptions regarding the role of smoking in lung cancer among blue-collar workers, more than $10 \%$ of the cases cannot be explained by smoking.

Finally, it is important to realize that the seemingly high estimates indicating that about one-quarter of the lung cancer burden is related to occupational exposures are actually in accordance with the general opinion that smoking is responsible for some $80 \%$ or even more of the risk for lung cancer. The reason is due to the likely or known interaction between smoking and many occu- pational exposures. Although rarely discussed, this interaction should also be kept in mind when the substantial risk of lung cancer from smoking is considered. Furthermore, for successful prevention, it is important to avoid the additive view, which implies that the high risk from smoking essentially rules out the need to remedy the lung cancer burden attributable to occupational and other factors.

It should also be remembered that the decreasing lung cancer rates that are now appearing for men in several countries may depend not only on the cessation of smoking, but also on improved work environments, especially the reduction of the use of asbestos. In Sweden, asbestos began to disappear from workplaces in the early 1980s and was almost completely gone by the early 1990s, although some asbestos-containing brake linings were produced until 1997. The import of asbestos dropped dramatically as early as the late 1970s, from around 18000 tonnes to less than 2000 tonnes. Inasmuch as the incidence rates (2) underlying my estimates were derived from a long period of time, any effects of declining asbestos exposure would have been accounted for. It is clear also that the occupational burden of lung cancer can be expected to decrease in the future, not only due to reduced exposure to asbestos, but also due to the reduction of other hazardous agents.

\section{References}

1. Nurminen M, Karjalainen A. Epidemiologic estimate of the proportion of fatalities related to occupational factors in Finland. Scand J Work Environ Health 2001;27:161-213.

2. Andersen A, Barlow L, Engeland A, Kjaerheim K, Lynge E, Pukkala E. Work-related cancer in the Nordic countries. Scand J Work Environ Health 1999;25 suppl 2:1-116.

3. Hammond EC, Selikoff IJ, Seidman H. Asbestos exposure, cigarette smoking and death rates. Ann NY Acad Sci 1979; 330:473-90.

4. Pershagen G, Wall S, Taube A, Linnman, L. On the interaction between occupational arsenic exposure and smoking and its relationship to lung cancer. Scand J Work Environ Health 1981;7:302-9.

5. Committee of biological effects of ionizing radiations, US National Research Council, BEIR IV. Health risk of radon and other internally deposited alpha-emitters. Washington (DC): National Academy Press, 1988.

6. Axelson O. Aspects on confounding in occupational health epidemiology [letter to the editor]. Scand J Work Environ Health 1978;4:98-102.

7. Dave SK, Edling C, Jacobsson P, Axelson O. Occupation, smoking and lung cancer. Br J Ind Med 1988;45:790-2.

8. Pershagen G, Akerblom G, Axelson O, Clavensjö B, Damber L, Desai G, et al. Residential radon exposure and lung cancer in Sweden. New Engl J Med 1994;330:159-64.

9. Carstensen JM, Pershagen G, Eklund G. Smoking-adjusted incidence of lung cancer among Swedish men in different occupations. Int J Epidemiol 1988;17:753-8. 
10. Nordlund LA, Carstensen JM, Pershagen G. Are male and female smokers at equal risk of smoking-related cancer: evidence from a Swedish prospective study. Scand J Public Health 1999;27:56-62.

11. Zang EA, Wynder EL. Differences in lung cancer risk between men and women: examination of the evidence. J Natl Cancer Inst 1996;88:183-92.

12. Statistics Sweden. Rökvanor i Sverige: en postenkätundersökning vären 1963 [Smoking habits in Sweden: a mail survey — spring 1963]. Stockholm: Statistiska Centralbyran, 1965.

13. Forastiere F, Perucci CA, Arca' M, Axelson O. Indirect estimation of lung cancer death rates in Italy not attributable to active smoking. Epidemiology 1993;4:502-10.

14. Kjuus H, Langård S, Skærven R. A case-referent study of lung cancer, occupational exposures and smoking, III: etiologic fraction of occupational exposures. Scand J Work Environ Health 1986;12:210-5.

15. Kvale G, Bjelke E, Heuch I. Occupational exposure and lung cancer risk. Int J Cancer 1986;37:185-93.

16. Dreyer L, Andersen A, Pukkala E. Avoidable cancers in the Nordic countries: occupation. APMIS Suppl 1997;76:68-79.

17. Dreyer L, Winther JF, Pukkala E, Andersen A. Avoidable cancers in the Nordic countries: tobacco smoking. APMIS Suppl 1997;76:9-47.

18. Doll R, Peto R. The causes of cancer: quantitative estimates of avoidable risks of cancer in the United States today. J Natl Cancer I 1981;66:1192-308.

19. Damber L, Larsson G. Occupation and male lung cancer: a case-control study in northern Sweden. Br J Ind Med 1987;44:446-53.

20. Pastorino U, Berrino F, Gervasio A, Pesenti V, Riboli E, Crosignani P. Proportion of lung cancer due to occupation. Int J Cancer 1984;33:231-7.

21. Ronco G, Ciccone G, Mirabelli D, Troia B, Vineis P. Occupation and lung cancer in two industrialized areas of northern Italy. Int J Cancer 1988;41:354-8.

22. Gustavsson P, Jakobsson R, Nyberg F, Pershagen G, Jarup L, Scheele P. Occupational exposure and lung cancer risk: a population-based case-referent study in Sweden. Am J Epidemiol 2000;52:32-40.

23. Kunst AE, Groenhof F, Mackenbach JP, Health EW. Occupational class and cause specific mortality in middle aged men in 11 European countries: comparison of population based studies: EU working group on socioeconomic inequalities in health. BMJ 1998;316:1636-42

24. Asp S. Confounding by variable smoking habits in different occupational groups. Scand J Work Environ Health 1984; $10: 325-6$.

Received for publication: 10 May 2001 